\title{
RNA-transfected dendritic cells in cancer immunotherapy
}

\author{
Duane A. Mitchell and Smita K. Nair \\ Center for Cellular and Genetic Therapies, Department of Surgery, Duke University Medical Center, Durham, North Carolina, USA \\ Address correspondence to: Smita K. Nair, Center for Cellular and Genetic Therapies, Department of Surgery, \\ Duke University Medical Center, Box 2601, Durham, North Carolina 27710, USA. \\ Phone: (919) 681-2180; Fax: (919) 681-7970; E-mail: s.nair@cgct.duke.edu.
}

The field of cancer immunotherapy has been recently invigorated by the discovery that vaccination with dendritic cells (DCs) pulsed with tumor antigens is a potent strategy to elicit protective immunity in tumor-bearing animals. The recognition that the cellular arm of the immune response is best equipped to recognize tumor cells as foreign and to lead to their eradication has shifted the emphasis in vaccine development. Vaccines that induce cellular responses, especially by the $\mathrm{CD}^{+}$cytotoxic $\mathrm{T}$ lymphocyte (CTL) arm of the immune system, are now favored over those that activate humoral immunity. At the same time, DCs have emerged as the most potent antigen-presenting cells (APCs) for eliciting antitumor CTLs. DCs can be generated from cancer patients by culturing adherent PBMCs from the patients for 5-7 days in the presence of cytokines (1).

The major research effort in many labs involves the choice of tumor antigen with which to load these DCs. The issues being addressed are, first, the composition of the antigen, whether a defined tumor antigen or an unfractionated mixture of tumor-derived antigens; and second, the form in which the antigen should be presented, whether as a polypeptide or a nucleic acid. Here, we focus on the use of RNA-transfected DCs in cancer immunotherapy, reviewing current data on the transfection of DCs with RNAs encoding either defined or unfractionated tumor antigens. We also consider the advantages and disadvantages of employing RNA transfection in loading DCs with tumor antigens and the merits of RNA transfection in situations where a low level of transiently expressed protein is sufficient to allow antigen presentation.

Nonviral versus viral gene transfer

The desired outcome of a DC vaccine is to elicit an immune response against antigens that are expressed endogenously within or loaded exogenously onto the DCs (2). For several reasons, expression within DCs of proteins other than the relevant tumor antigens may interfere with generating a potent antitumor immune response. First, a poorly understood but well-documented immunological phenomenon called immunodominance may occur, whereby other antigenic proteins, such as viral antigens, mask or suppress the response to the less potent tumor antigens (3). Second, activated CTLs may recognize and kill antigen-presenting DCs (4). In particular, DCs expressing both viral and tumor antigens may be eliminated by virusspecific $T$ cells before they can activate a tumor-specific response. If a viral vector is used to deliver the tumor antigen, this elimination effect would be amplified with subsequent immunizations, as it is likely that the virus-specific T-cell pool would be expanded with each vaccination. Hence, it seems desirable to develop methods of DC transfection that lead to the expression of only those proteins toward which an immune response is desired. It should be noted that while many viral vectors have been developed which limit the expression of viral proteins in the target cells, DCs are very efficient at processing and presenting proteins of inactivated viruses that they endocytose, so the potential problem of immunodominance may be unavoidable even with these improved vectors (5).

The use of nucleic acids in DC loading

DCs must be loaded with tumor antigens in order to elicit an immune response against cancer cells in vaccinated patients. These antigens can be in the form of peptides, cellular extracts from tumor cells, apoptotic bodies, purified proteins, or nucleic acids (6-10). These antigens are then processed and displayed on the surface of the DCs as defined antigenic peptides, which lead to the activation of tumor-specific CTLs. The method of choice for loading DCs with tumor antigens would be the one that leads to the most efficient display of antigenic tumor-derived peptides in a manner that elicits the most potent antitumor T-cell immune response. For reasons discussed below, transfection with nucleic acid may be preferred for this purpose.

The number of known tumor antigens - proteins expressed specifically in malignant cells against which the immune system can mount a response - is small, even for melanoma, which is the best-defined type of tumor in this respect. In addition, because patients whose tumors have recurred after primary therapy often present with small, disseminated metastases that are difficult to culture, the amount of tumor tissue available is often very limited (11). Thus, methods of DC loading that depend on knowing the structure 
of specific tumor antigens or that rely on the availability of abundant tumor tissue (cellular extracts or apoptotic bodies) are applicable to very few cancer patients. While continued advances in antigen discovery and tissue culture technique may alleviate these difficulties and allow broader use of immunotherapies based on defined antigens, alternative approaches will be needed for the foreseeable future. Nucleic acid transfection, which relies on well-characterized methods of cDNA cloning and amplification, allows for the antigenic content of tumors to be isolated from a small number of tumor cells (12). In fact, a cDNA library can be made from even a single cell, allowing the content of tumor cells to be isolated and amplified from nearly any cancer patient.

The use of nucleic acid templates for the expression of tumor-derived antigens allows for the expression of the antigens as full-length proteins within DCs. This allows the antigen-processing machinery of the patients' own DCs to process these proteins and display the peptides on their surface. A reasonable assumption is that the most relevant peptides are presented to the patient's immune system. Vaccine strategies based on synthetic peptides or proteins require knowledge of the relevant peptides for that patient pool and assume that the peptides chosen will be the ones best recognized by the immune system of that patient. When DCs are transfected with the tumor antigens, the patient's own immune system makes that choice. Thus, for both immunological and practical considerations, nucleic acid transfection of DCs offers several advantages over other described methods.

\section{RNA versus DNA transfection}

DNA vectors have been used far more frequently than RNA for gene expression in DCs and other cell types (13-15). Historically, DNA has been used for most transfections. It is more stable than RNA and can be produced in large quantities, and the template for expression of the cloned gene can be modified to regulate expression of the gene. Although reports of cancer immunotherapy using DCs transfected with nonviral DNA vectors are few, it appears that these DCs can elicit antitumor immune responses (15-17). These vaccines are believed to work by direct transfection of DCs, such as the Langerhans cells within the skin.

RNA transfection of DCs may be advantageous in several respects. The mRNA content of tumor cells can be directly isolated from tumors (when sufficient material is available) and can be transfected into DCs with no intervening cloning steps. In the more common scenario, amplification of the mRNA content can be achieved using RT-PCR. If libraries are devised so that a bacteriophage RNA polymerase promoter is present in the $5^{\prime}$ end of each CDNA, an unlimited supply of in vitro transcribed mRNA is available without the need for cloning or the subsequent removal of bacterial con- taminants. Moreover, transfected RNA need only reach the cytoplasm of DCs, whereas DNA requires entry into the nucleus and subsequent transcription to generate the encoded message.

A potentially significant advantage of using RNAencoded antigens is safety. The half-life of stable mRNA species in the mammalian cell is less than 24 hours, while unintegrated DNA can persist and function in nondividing cells for months. Vaccination with total tumor-derived antigens in the form of DNA would be of greater concern, especially if tumor antigens that are mechanistically implicated in tumorigenesis, such as the products of the human papilloma virus E6 or E7 genes, were to be introduced into DCs.

Another major advantage of RNA transfection is its ability to express several tumor-derived genes within the DCs. Multiple mRNA species can be transfected into the target cell, leading to the translation of several different gene products within the same cell. With DNA transfection, in contrast, only one or a few copies of a DNA vector will usually be incorporated into each cell, therefore limiting the expression within each DC to a few proteins. To incorporate a desired gene in the form of RNA only requires adding that species to the mixture to be transfected; DNA technology would require additional cloning steps.

There are also some drawbacks to the use of RNA for transfection. RNA is a more labile molecule than DNA. The half-life of RNA is approximately 5 hours in serum-free media but is measured in minutes when serum is present. However, although it might be expected that the levels of expressed protein following RNA transfection would be less than those achieved by DNA transfection, studies that have compared the two show comparable levels of reporter gene activity (18). No such comparison between DNA and RNA transfection has been reported in DCs, but we have noted efficient expression of a green fluorescent protein reporter gene following RNA transfection of transformed cell lines and much lower expression in comparably treated DCs (S.K. Nair, unpublished observations). Whether DCs synthesize less protein or degrade the expressed marker more rapidly than the other cells is not known, but other transfection methods can lead to higher levels of protein expression in DCs (19).

The low level of intact protein expression achieved by RNA transfection would clearly be a limitation if the intent were to modify the function of the target cell with the expressed protein. However, when the explicit goal is to elucidate a potent immune response against the transfected antigens, expression at a low level offers a theoretical, but perhaps not insignificant, advantage: The effectiveness of a $\mathrm{T}$ cell-mediated immune response is greatly influenced by how readily the $\mathrm{T}$ cells are able to recognize and kill their targets and how abundantly those target peptides are expressed. The overall combination of T-cell receptor affinity and 
abundance of antigenic peptide on target cells is referred to as the avidity of the immune response. If a given antigenic peptide is relatively scarce on the surface of target cells, as is likely the case for tumor antigens on cancer cells, then only $\mathrm{T}$ cells with high-affinity receptors will allow a response of sufficient avidity to permit the killing of the target cell. When DCs present an abundant level of antigenic peptides to the immune system, the $T$ cells activated can be of low affinity and thus mask the activation of the high-affinity T cells, which may generate a population of $T$ cells that can kill targets that carry large amounts of the peptide on their surface, but that cannot kill the relevant tumor cells. Conversely, when a low level of antigenic peptides is presented by DCs, only those T cells with a high-affinity recognition are activated, thus skewing the response toward T cells that can better recognize the tumor cells. Such skewing toward high- or low-affinity $\mathrm{T}$ cells, depending on the amount of peptide on the APC surface, has indeed been observed (20). Based on this consideration, the low level of protein expression in RNA transfected DCs may be advantageous, provided that expression levels are sufficient to generate a T-cell response. In studies using the model tumor antigen carcinoembryonic antigen (CEA), we showed that DCs transfected with CEA RNA are comparable to peptidepulsed DCs in stimulating $T$ cells against the defined peptide epitope, and more effective than peptidepulsed DCs in stimulating $T$ cells against tumor cells expressing CEA (21). While we cannot directly attribute the greater effectiveness of RNA-transfected DCs to the activation of higher affinity $\mathrm{T}$ cells, we conclude that the lower expression of antigenic protein does not appear to limit the efficacy of the DCs.

\section{Methods of RNA transfection}

The delivery of RNA into target cells was initially described in studies that investigated the use of RNA genome viruses (22). Most recent studies exploring the delivery of RNA to target cells have used RNA/liposome complexes to achieve transfection (23-25). Experience with DNA transfection has taught us that the efficiency of gene transfer is greatly influenced by the choice of lipid vehicle, the ratio of nucleic acid to lipid, the concentration versus toxicity of the complexes, and the cell type one desires to transfect. Few comparative studies have been published for transfection of RNA, but $\mathrm{Lu}$ et al. report that RNA/DOTAP complexes achieve the strongest expression of a reporter gene mRNA in a human breast cancer line (23) and that expression is affected by both the choice of lipid and the lipid/RNA ratio. Glenn et al. (24) have also explored the use of liposomes incorporating proteins as a means to target delivery of RNA/liposome complexes to specific cells (24). Finally, gene gun (biolistic) delivery of mRNA into cultured cells can be used for transgene expression to elicit immunity (26).
Incorporation of the $5^{\prime}$ and $3^{\prime}$ untranslated regions of $\beta$-globin mRNA greatly stabilizes RNA transfected into cells and leads to over a 1,000-fold increase in reporter gene expression in transfected cells (25). We have also found that lengthening of the polyA tail in the mRNA transcripts increases expression of GFP in target cells (S.K. Nair, unpublished observations). While it has been reported that RNA transfection in vivo requires a lipid carrier, we have found that human DCs can be transfected in vitro using naked RNA (21). The ability to transfect DCs efficiently without lipid depends on exposing them to the RNA when they are immature, a stage when they take up exogenous material avidly. We have found that DCs transfected with naked RNA were comparable to those transfected with RNA/lipid complexes in the stimulation of antigenspecific CTL responses but required that more RNA be used. Thus, the cloning, mRNA synthesis, and transfection strategy one chooses when designing an RNAbased vaccine can greatly influence the expression pattern and probably the immunological outcome.

\section{RNA-transfected DCs as cancer vaccines}

Strategies proposed for cancer vaccines are numerous, but resources for testing them are limited. Thus, the decision of whether to advance a given approach to clinical trials must rest on evidence for its potential advantages, wide-scale applicability, and efficacy in defined animal models. The greatest advantage of the use of RNA-transfected DCs is the ability to extend this approach to a wide number of patients who previously were excluded from clinical trials due to limitations in obtaining tumor material. Building on existing technologies in molecular biology, we have found that tumor mRNA can be isolated from a very small number of tumor cells, amplified, and used without loss of biological function (12). For instance, RNA isolated from tumor cells that were microdissected from pathology slides can be amplified and used to generate an antitumor immune response when transfected into DCs. The methodology employed for amplifying the mRNA greatly influences the efficiency of amplification as well as the fidelity of the RNA obtained. We have found that using a primer in the reverse transcription reaction that generates a long polyA tail in mRNA product leads to better CTL responses (12).

The efficacy of RNA transfection in eliciting immunity in vivo has been seen in several mouse studies, in which potent CTL responses developed in animals immunized with mRNA/lipid complexes (27-31). These responses were comparable to those achieved by infection with live virus, indicating a physiologically significant level of immunity achieved by vaccination with RNA. In most cases, DCs transfected with RNA have proved superior to peptide-pulsed DCs in eliciting CTL responses against either peptide-pulsed experimental targets or intact tumor cells $(10,12,21)$. Fur- 
thermore, in tumor challenge experiments and stringent animal models of experimental metastasis, DCs transfected with in vitro transcribed RNA or total tumor RNA elicit protective antitumor immune responses $(10,12,31)$. Zhang et al. demonstrated the efficacy of tumor RNA-pulsed DCs in the 3LL lung carcinoma model and demonstrated that they could enhance the antitumor effect of their vaccine by expressing the chemokine lymphotactin in the DCs (30). Fortunately, no autoimmune responses have been detected in mice vaccinated with DCs transfected with total tumor RNA.

The efficacy of in vivo vaccines that aim for T-cell immunity probably depends on the presentation of the relevant antigens by DCs. It remains to be seen whether strategies delivering antigen ex vivo into cultured DCs or those based on direct immunization in vivo will be the most effective at eliciting potent antitumor immune responses.

\section{Future directions}

A major limitation to immunotherapy development for malignancy at present lies in the lack of intermediate markers of success. Because in most cases the relevant antigen is unknown and little tumor tissue is available, patients' immune responses to vaccination can now be evaluated only by following the clinical regression of their tumors. While this is one of the clinical endpoints in which we are most interested (the other being survival), it would be useful to monitor several immunological endpoints by sampling the patient's serum and measuring T-cell responses against the tumor cell targets. Transfecting DCs with mRNA encoding the antigenic content of the tumor cell has allowed us to devise tests for immune responses despite our ignorance of specific tumor targets. We now can engineer "tumorcell equivalents" or DCs transfected with tumor mRNA as targets to monitor the patient's response against the administered vaccine. We find that the cytotoxic $\mathrm{T}$ lymphocytes elicited in a cancer patient by RNA-transfected DCs can lyse the patient's own tumor cells, as well as DCs transfected with the relevant tumor RNA, but not cells transfected with unrelated tumor RNA (S.K. Nair et al., unpublished observations). These studies suggest that RNA-transfected DCs can be used as surrogate targets to reliably measure the immune response that will be directed against the real targets, the patient's own tumor cells.

While RNA transfection is now adequate to direct low levels of protein expression and allow for antigen processing in DCs, this tool is not yet able to modify cellular function. To this end, RNA templates might be modified to improve stability and also to redirect processing of the protein. Thus, incorporating a lysosomal targeting sequence into the CEA transcripts enhances CEA-specific Th cell responses, possibly because the expressed protein is redirected to the processing path- way responsible for activating Th cells (21). Incorporation of Th cells in the vaccine strategy should improve CTL responses in patients. In addition, since cell-specific targeting of DNA-lipid complexes can enhance gene expression, it is likely that a similar approach adapted to the RNA platform will improve delivery of mRNA to DCs.

Systematic searches for human tumor antigens recognized by $\mathrm{T}$ cells have generally been limited to studying melanoma. Melanoma cells, unlike those in many other types of cancers, can be established in culture. Furthermore, melanoma patients exhibit a weak but specific CTL response which can be expanded ex vivo. RNA transfection offers a means to expand the scope of vaccination to other classes of tumors by providing an effective and readily measured method for generating a tumor-specific T-cell response and greatly reducing the amount of tumor tissue needed for antigen isolation. Because tumor mRNA-transfected DCs are potent stimulators of T-cell immunity in vitro and in vivo $(10,12,21,31)$ and can function as targets in CTL cytotoxicity assays, they offer a practical substitute for tumor cells in assays to test the immunological effects of specific antigens.

In summary, the use of DCs transfected with RNA for tumor immunotherapy is an effective vaccination platform. The versatility and safety of RNA-based vaccines warrants further exploration of this strategy. We hope that, at the very least, more investigators will have acquired experience with RNA-based DC vaccines and that this approach will have been attempted in a greater variety of experimental models and clinical settings by the time the next review on this topic is published.

\footnotetext{
1. Romani, N., et al. 1995. Proliferating dendritic cell progenitors in human blood. J. Exp. Med. 180:83-93.

2. Steinman, R.H. 1996. Dendritic cells and immune-based therapies. Exp. Hematol. 24:859-862.

3. Pion, S., Christianson, G.J., Fontaine, P., Roopenian, D.C., and Perreault, C. 1999. Shaping the repertoire of cytotoxic T lymphocyte responses: explanation for the immunodominance effect whereby cytotoxic $\mathrm{T}$ lymphocytes specific for immunodominant antigens prevent recognition of nondominant antigens. Blood. 93:952-962.

4. Ingulli, E., Mondino, A., Khoruts, A., and Jenkins, M.K. 1997. In vivo detection of dendritic cell antigen presentation to CD4+ T cells. J. Exp. Med. 185:2133-2141.

5. Bender, A., Bui, L.K., Feldman, M.A., Larsson, M., and Bhardwaj, N. 1995. Inactivated influenza virus, when presented on dendritic cells, elicits human CD8+ cytolytic T cell responses. J. Exp. Med. 182:1663-1671.

6. Mayordomo, J.I., et al. 1995. Bone marrow-derived dendritic cells pulsed with synthetic tumor peptides elicit protective and therapeutic antitumor immunity. Nat. Med. 1:1297-1302.

7. Nestle, F.O., et al. 1998. Vaccination of melanoma patients with peptideor tumor lysate-pulsed dendritic cells. Nat. Med. 4:328-332.

8. Albert, M.L., et al. 1998. Immature dendritic cells phagocytose apoptotic cells via $\alpha_{v} \beta_{5}$ and CD36, and cross-present antigens to cytotoxic T lymphocytes. J. Exp. Med. 188:1359-1368.

9. Paglia, P., Chiodoni, C., Rodolfo, M., and Colombo, M.P. 1996. Murine dendritic cells loaded in vitro with soluble protein prime cytotoxic T-lymphocytes against tumor antigen in vivo. J. Exp. Med. 183:317-322.

10. Boczkowski, D., Nair, S.K., Snyder, D., and Gilboa, E. 1996. Dendritic cells pulsed with RNA are potent antigen-presenting cells in vitro and in vivo. J. Exp. Med. 184:465-472.
} 
11. Gilboa, E. 1999. The makings of a tumor rejection antigen. Immunity. 11:263-270.

12. Boczkowski, D., Nair, S.K., Nam, J., Lyerly, H.K., and Gilboa, E. 2000. Induction of tumor immunity and cytotoxic $\mathrm{T}$ lymphocyte responses using dendritic cells transfected with messenger RNA amplified from tumor cells. Cancer Res. 60:1028-1034.

13. Manickan, E., Sivadasan, K., Rouse, R.J.D., Yu, Z., and Rouse, B.T. 1997. Enhancement of immune response to naked DNA vaccine by immunization with transfected dendritic cells. J. Lenkoc. Biol. 61:125-132.

14. Arthur, J.F., et al. 1997. A comparison of gene transfer methods in human dendritic cells. Cancer Gene Ther. 4:17-25.

15. Tuting, T., Deleo, A.B., Lotze, M.T., and Storkus, W.J. 1997. Genetically modified bone marrow-derived dendritic cells expressing tumor-associated viral or "self" antigens induce antitumor immunity in vivo. Eur. J. Immunol. 27:2702-2707.

16. Condon, C., Watkins, S.C., Celluzzi, C.M., Thompson, K., and Falo, L.D. 1996. DNA-based immunization by in vivo transfection of dendritic cells. Nat. Med. 2:1122-1127.

17. Pardoll, D.M., and Beckerleg, A.M. 1995. Exposing the immunology of naked DNA Vaccines. Immunity. 3:165-169.

18. Yarovoi, S.V., et al. 1996. In vitro sensitization of the B16 murine melanoma cells to ganciclovir by different RNA and plasmid DNA constructions encoding HSVtk. Gene Ther. 3:913-918.

19. Di Nicola, M., et al. 1998. Gene transfer into human dendritic antigenpresenting cells by vaccinia virus and adenovirus vectors. Cancer Gene Ther. 5:350-356.

20. Vitiello, A., et al. 1997. Comparison of cytotoxic T lymphocyte responses induced by peptide or DNA immunization: implications on immunogenicity and immunodominance. Eur. J. Immunol. 27:671-678.

21. Nair, S.K., et al. 1998. Induction of primary carcinoembryonic antigen (CEA)-specific cytotoxic T lymphocytes in vitro using human dendritic cells transfected with RNA. Nat. Biotechnol. 16:364-369.

22. Ahlquist, P., French, R., Janda, M., and Loesch-Fries, L.S. 1984. Multicomponent RNA plant virus infection derived from cloned viral cDNA. Proc. Natl. Acad. Sci. USA. 81:7066-7070.

23. Lu, D., Benjamin, R., Kim, M., Conry, R.M., and Curiel, D.T. 1994. Optimization of methods to achieve mRNA-mediated transfection of tumor cells in vitro and in vivo employing cationic liposome vectors. Cancer Gene Ther. 1:245-252.

24. Glenn, J.S., Ellens, H., and White, J.M. 1993. Delivery of liposome-encapsulated RNA to cells expressing influenza virus hemagglutinin. Methods Enzymol. 221:327-339.

25. Malone, R.W., Felgner, P.L., and Verma, I.M. 1989. Cationic liposomemediated RNA transfection. Proc. Natl. Acad. Sci. USA. 86:6077-6081.

26. Qui, P., Ziegelhoffer, P., Sun, J., and Yang, N.S. 1996. Gene gun delivery of mRNA in situ results in efficient transgene expression and genetic immunization. Gene Ther. 3:262-268.

27. Hoerr, I., Obst, R., Rammensee, H.-G., and Jung, G. 2000. In vivo application of RNA leads to induction of specific cytotoxic T lymphocytes and antibodies. Eur. J. Immunol. 30:1-7.

28. Martinon, F., et al. 1993. Induction of virus-specific cytotoxic T lymphocytes in vivo by liposome-entrapped mRNA. Eur. J. Immunol. 23:1719-1722.

29. Zhou, W.Z., et al. 1999. RNA melanoma vaccine: induction of antitumor immunity by human glycoprotein 100 mRNA immunization. Hum. Gene Ther. 10:2719-2724.

30. Zhang, W., et al. 1999. Enhanced therapeutic efficacy of tumor RNApulsed dendritic cells after genetic modification with lymphotactin. Hum. Gene Ther. 10:1151-1161.

31. Ashley, D.M., et al. 1997. Bone marrow-generated dendritic cells pulsed with tumor extracts or tumor RNA induce antitumor immunity against central nervous system tumors. J. Exp. Med. 186:1177-1182. 